\section{Mensch-Technik-Interaktivität mit Servicerobotern}

Ansatzpunkte für eine techniksoziologisch informierte TA der Robotik

von Martin Meister, TU Berlin

Intelligente Roboter stehen als Serviceroboter an der Schwelle zum Einzug in unseren Alltag. Das ist eine potenziell folgenreiche Entwicklung, die sowohl eine Beteiligung sozialwissenschaftlicher Begleitforschung an diesem Prozess als auch eine profunde Technikfolgenabschätzung auf den Plan rufen sollte. Der Diskurs zu dieser Frage ist aber durch zwei sich unvermittelt gegenüberstehende Lager gekennzeichnet, denen gemeinsam ist, dass sie von übergeneralisierten Annahmen über „den Menschen" ausgehen. In diesem Beitrag wird auf techniksoziologischer Grundlage für einen balancierten und empirisch offenen Ansatz plädiert und für empirische Untersuchungen der MenschRoboter-Interaktivität geworben. Auf der Entwicklerseite gibt es einige Forschungs- und Diskussionsstränge, die in besonderer Weise anschlussfähig scheinen für eine techniksoziologisch informierte TA der Robotik und Kooperationschancen eröffnen.

\section{Einleitung}

Viele Forschende in der „Neuen Robotik“ sind der Überzeugung, dass sich ihre autonomen Maschinen soweit entwickelt haben, dass sie nunmehr in der Lage sind, in sich verändernden Umgebungen zu navigieren, sich an diese Umgebungen anzupassen und darin für menschliche Nutzer sinnvolle Dinge selbständig zu verrichten. Die Herausgeber des aktuellen Handbuches beschreiben die zentrale Herausforderung der Robotik wie folgt:

\footnotetext{
„Reaching for the human frontier, robotics is vigorously engaged in the growing challenges of new emerging domains. Interacting, exploring, and working with humans, the new generation of robots will increasingly touch people and their lives. The credible prospect of practical robots among humans is the result of the scientific endeavor of a half a century of robotic developments that established robotics
}

as a modern scientific discipline." (Siciliano; Khatib 2008, S. XVII)

Wenn Serviceroboter in unseren Alltag Einzug halten als Helfer, Führer und Partner, dann ist das eine folgenreiche Entwicklung, die sowohl nach der Beteiligung sozialwissenschaftlicher Begleitforschung in diesem Prozess als auch nach einer profunden Technikfolgenabschätzung rufen sollte. Dieser Ruf ist allerdings noch nicht laut geworden, was daran liegt, dass der Diskurs bislang durch zwei sich unvermittelt gegenüberstehende Lager gekennzeichnet ist, denen gemeinsam ist, dass sie von übergeneralisierten Annahmen über „den Menschen“ ausgehen. Dabei erscheint den Ingenieurwissenschaften und der Forschung zu Künstlicher Intelligenz (KI) auf der einen Seite „der Mensch“ als ultimative Herausforderung. Den Geistes- und Sozialwissenschaftlern auf der anderen Seite erscheint, insbesondere wenn sie sich für „Roboethics“ einsetzen, dagegen der Roboter als Gefahr.

In diesem Beitrag wird auf techniksoziologischer Grundlage für einen balancierten Ansatz plädiert und für empirische Untersuchungen der Mensch-Roboter-Interaktivität. Das könnte dazu beitragen, herauszufinden, was „ganz normale Menschen" akzeptabel, nützlich oder sogar attraktiv an avancierten Robotern in ihren Alltagsumgebungen finden, und wie (und in welchen Grenzen) ein wechselseitiger Anpassungsprozess zwischen „schlauen“ Robotern und ,ganz gewöhnlichen" Menschen möglich und sinnvoll ist.

Nach einer kurzen Skizze der Ausgangslage werden drei konkrete Anknüpfungspunkte herausgearbeitet, an denen eine techniksoziologisch informierte TA sich produktiv in die Roboterforschung einbringen könnte.

\section{Die durch Lagerbildung blockierte wechselseitige Bezugnahme}

Aus der Perspektive der Forschenden in der Robotik stellen menschliche Alltagsumgebungen die schwierigste Art von Domäne dar. Anders als Industrieroboter, die die immer gleichen Abläufe in einer vollständig definierten Umgebung vollziehen, und anders als Feldroboter, die weit weg von Menschen operieren, sind Serviceroboter dafür ausgelegt, in Kopräsenz mit einem verstören- 
den und in seinen Aktionen sehr schwer vorhersehbaren Faktor zu operieren: ganz gewöhnlichen menschlichen Wesen. ${ }^{1}$ Es ist schwer zu sagen, welchen formal definierbaren Regeln sie in den Situationen ihres Alltags folgen. Es wäre außerdem damit zu rechnen, dass sie sich häufig an solche Regeln, ob absichtlich oder nicht, letztlich nicht hielten. Jede dieser alltäglichen Handlungen stellt enorme Herausforderungen für einen Roboter dar - insbesondere für seine Selbstlokalisierung und Navigation, seine Modelle für Steuerung und Entscheidung sowie das Design seiner Sensoren und Interfaces, um nur einige der technischen Probleme zu nennen. Und all diese Einzelprobleme müssen in einer einzigen Plattform gleichzeitig und in „real time“ gelöst werden die eigentliche Herausforderung der Robotik.

Um einen Roboter in einer solchen, wie es im Feld heißt, „komplexen“ Umgebung operabel zu machen, müssen die Menschen irgendwie bei der Modellierung der Maschine berücksichtigt werden. Es gibt dafür verschiedene Ansätze auf Entwicklerseite, denen gemeinsam ist, dass sie alltägliche menschliche Aktivität als eine Ressource zu nutzen versuchen, um eine herausfordernde Umgebung zu verstehen und formal abzubilden. Und sie sind allesamt von der Annahme geleitet, dass ein Roboter, der in Aussehen, Interface oder Verhaltensweisen einem Menschen ähnelt, von potenziellen Nutzern auch besser bedient und eher akzeptiert werden kann. Dabei folgen fast alle Entwickler der „I-Methodology“ (Akrich 1995): Die Entwickler imaginieren sich einfach selbst als ideale Nutzer, d. h. das Risiko von produktiven Irritationen, die sich aus der empirischen Untersuchung der Wünsche, Visionen und praktischen Kreativität der Nutzer ergeben könnten, wird ausgeklammert.

Während also von Entwicklerseite die „Human Frontier" als ultimative Herausforderung für die Maschinen aufgefasst wird, verhält es sich bei den meisten geistes- und sozialwissenschaftlichen Beiträgen zur „Roboethics“ genau andersherum. Serviceroboter, die in den Alltag eindringen, überschreiten aus dieser Sicht eine rote Linie. Als geeignete Maßnahme wird etwa vorgeschlagen, Robotern moralische Prinzipien ,einzupflanzen“, und es wird ernstlich diskutiert, ab welchem Entwicklungsstadium ein Roboter für seine Aktionen moralisch verantwortlich ist (Wallach 2010). Alterna- tiv dazu gibt es in der „Roboethics“ auch den Ansatz $^{2}$, nicht die Roboter, sondern deren Entwickler als moralische Wesen zu adressieren, und an deren „professionelle Verantwortlichkeit" zu appellieren und Maßnahmen zu fordern, die diese stärken:

„Roboethics is not a veto or a prohibitionist eth-
ics. Its main lines of development are: the promo-
tion of culture and information; the permanent
education; a vigorous and straight public debate;
and the involvement in all these activities of the
young generations who are the actors of the fu-
ture." (Veruggion, Operto 2008, S. 1515)

In der Praxis konzentriert sich Roboethics allerdings darauf, eine umfassende Taxonomie möglicher Gefährdungen „des Menschen“ aufzustellen, und diese Taxonomie dann nach Anwendungsgebieten zu differenzieren. Die Liste möglicher Gefahren ist sehr lang, und umfasst zunächst die aus der TA- und Ethikdiskussion um Informationstechnologien vertrauten Themen: Fragen der Zuverlässigkeit, Schutz der Privatsphäre, Datenschutz, „Dual Use“, „Digital Divide“ und Ersetzung menschlicher Arbeitskräfte. Zusätzlich kommen Gefahren in den Blick, die sich aus der schlichten physischen Nähe der Roboter zu Menschen ergeben - Probleme, die in den Laborplattformen durch Abstandssensoren und den berühmten roten Abschaltknopf gelöst werden, aber für Alltagssituationen spezifiziert werden müssen. Eine dritte Kategorie bilden ,psychologische Probleme“, womit etwa ,deviations in human emotions, problems of attachment (...) fears, panic (...) feeling of subordination towards robots" (Veruggio; Operto 2008, S. 1512) gemeint sind. Diese Probleme werden letztlich auf eine gleichsam ontologische „confusion between the artificial and the real“" (ebenda) zurückgeführt, durch die „der Mensch“ ganz prinzipiell überfordert wird.

\section{Für eine balanciertere und empirisch offene Sicht des Mensch-Roboter-Verhältnisses}

Die Frontstellung zwischen dem Menschen als Herausforderung und dem Roboter als Gefahr blockiert nicht nur den Diskurs, sondern jedwede Form eines produktiven Austausches zwischen den Ingenieurswissenschaften und den Sozialund Geisteswissenschaften. Die neuere Techniksoziologie bietet einen Ansatzpunkt, diese Kluft 
zu verringern, und zwar sowohl von den konzeptionellen Grundlagen her als auch in Bezug auf die praktischen Konsequenzen für eine TA der neuen Robotik.

Zunächst sollte nicht von „Interaktion“ mit Robotern, also mit Maschinen (auch nicht mit „schlauen“"Maschinen) gesprochen werden, denn diese Begrifflichkeit evoziert natürlich den Vergleich und führt zu der unfruchtbaren KI-Frage, ob Maschinen denken oder, hier relevanter: handeln können (Rammert, Schulz-Schaeffer 2002). Mit dem Begriff der „Interaktivität" (Rammert 2008) ist im Kern gemeint, dass Prozesse zwischen zwei grundsätzlich unterschiedlichen Entitäten (manche KI-Forscher würden „Spezies“ sagen) untersucht werden sollten, wobei das Ergebnis aus diesem Prozess selbst hervorgeht.

Diese Konzeptualisierung hat Konsequenzen auf beiden Seiten: Soziologisch bedeutet das, den Begriff der Handlungsträgerschaft zu verteilen, d. h. dass das „Handeln-Können“ nicht nur Menschen vorbehalten wird, sondern die Handlungsträgerschaft zwischen Menschen, Maschinen und Programmen verteilt ist (Rammert 2008; Weyer 2008). Auf der Seite der Robotik bedeutete das, avancierte Roboter unter dem Gesichtspunkt der Interaktivität $\mathrm{zu}$ modellieren und ,intelligentes Roboterverhalten“" an der Fähigkeit auszurichten „to retrieve information through interaction with humans" (Weiss et al. 2010, S. 38) und weitergehend dann auch so ausgelegt werden müssten, dass sie Informationen von den Nutzern auch aktiv einfordern und sich daran anpassen. Es geht dann also um die Konzeptualisierung einer dreistelligen Relation: das Handeln der Menschen, die Aktionen des Roboters und die des Prozesses zwischen beiden $^{3}$, der dann als Lernen oder als wechselseitige Anpassung beschrieben werden kann.

Gegen eine übergeneralisierte Sicht auf ,den Menschen" können Techniksoziologie und die „Science and Technology Studies“ die zentrale Erkenntnis setzen, dass die „Passung“ relativ zum jeweiligen sozialen Bedarf zentral für den Erfolg technischer Gerätschaften ist. Dazu gehört auch das Wissen, dass verschiedene Nutzergruppen für sie neue Artefakte an ihre jeweiligen Bedürfnisse anpassen, oder diese Artefakte sogar kreativ verändern bis hin zur Neuerfindung in der Anwendung (s. Oudshoorn, Pinch 2007). Welche unter- schiedlichen Nutzergruppen Serviceroboter unter welchen Bedingungen akzeptieren und welche versuchen werden, sie konstruktiv anzupassen oder sogar technisch weiterzuentwickeln, kann aus dieser Sicht nur empirisch eruiert werden.

\section{Ansatzpunkte für Techniksoziologie und TA}

Es bleibt die Frage, wie diese techniksoziologische Perspektive über die Einbindung entsprechender Akteure nicht nur als kritischer Kommentar, sondern im konkreten F\&E-Prozess in Anschlag gebracht werden kann, etwa im Sinne des „Constructive Technology Assessment" oder des „Real-time Technology Assessment“, die darauf hinweisen, dass neue technologische Entwicklungen in ihren frühen Stadien konstruktiv beeinflusst werden müssen. ${ }^{4}$

An welchen Stellen könnte die techniksoziologisch informierte TA ihre Perspektive und ihre empirischen Forschungsansätze aussichtsreich in den Entwicklungsprozess einbringen? Das kann sie offensichtlich nicht im Bereich von ELSI (,ethical, legal and social issues"), denn diese Anstrengungen bleiben ein Add-on, das mit der eigentlichen Entwicklungsarbeit bestenfalls am Rande zu tun hat. Das liegt nicht daran, dass die Entwickler grundsätzliche Vorbehalte gegen ELSI-Studien hätten oder diese ablehnten, sondern weil deren Erträge für ihr Kerngeschäft einfach nicht wichtig sind. Es gibt aber aus meiner Sicht drei chancenreiche Anknüpfungspunkte, an denen die Techniksoziologie an die Praxis der Roboterforschung anschließen kann.

\section{Ansatzpunkt 1: Roboter als Verkörperung der großen Ideen der Kybernetik}

Für die „Neue Robotik“ ist unübersehbar, dass die „Prehistory of the New Artificial Intelligence“5 nahezu immer auf die Ursprünge in der klassischen Kybernetik der 1940er und 1950er Jahre verweist, wobei die aktuellen Roboterkreaturen als Verkörperung der großen Ideen der Kybernetik dargestellt werden: (1) die Idee, Menschen und Maschinen in einer einheitlichen Beschreibungssprache fassen zu können, nicht zuletzt um ihre enge Verzahnung angemessen fassen zu können; 
(2) die Notwendigkeit, höchst unterschiedliche Disziplinen zu einer gemeinsamen Anstrengung zusammenzufassen; (3) ein Verantwortungsbewusstsein der Wissenschaftler für die Konsequenzen ihrs Tuns, wie sie beispielsweise im Handbuch-Artikel zu Roboethics vorgetragen wird:

\begin{abstract}
„Even a restricted knowledge of cybernetics and computer science, from Wiener, to von Neumann, to Weizenbaum, will immediately and directly demonstrate that these scientists immediately took care of the ethical and social aspects of their discoveries and realizations, which marked the beginning of the field of computers and robotics. "“
\end{abstract}

Diese historische Referenz wird in zwei unterschiedlichen Versionen ins Spiel gebracht. In der dominanten Version wird die „Design Philosophy of Service Robots“ (Kawamura et al. 1996) als Modellierung einer „Mensch-MaschineSymbiose" gefasst, bei der die Menschen nur ein Element in einer übergreifenden Systemdynamik sind. Für die techniksoziologische Perspektive interessanter ist die zweite Version, die an eine Geschichte ,niedlicher kybernetischer Kreaturen" anschließt (Meister, Lettkemann 2004), deren komplexe Verhaltensweisen uns ,erfreuen und unterhalten" sollen (Fong et al. 2003, S. 161) und die als Vorbilder für die heutigen, avancierteren Roboter vorgestellt werden. Die entsprechende Designphilosophie liest sich wie folgt:

\begin{abstract}
„Humans and robots must be able to coordinate their actions so that they interact productively with each other. It is not appropriate (or even necessary) to make the robot as socially competent as possible. Rather, it is more important that the robot be compatible with the human's needs, that it matches application requirements; that it be understandable and believable, and that it provide the interactional support the human expects.“ (dies., S. 160f.)
\end{abstract}

Hier wird der Roboter in der Rückkopplungsschleife des menschlichen Alltags positioniert, und das technische Design des Roboters bzw. die entsprechenden Modellierungsentscheidungen werden an dieser Philosophie orientiert. Daher könnte diese Version der kybernetischen Traditionslinie einen geeigneten Anknüpfungspunkt für eine im oben genannten Sinne techniksoziologisch orientierte Perspektive darstellen - und zugleich einen Anknüpfungspunkt nicht nur für rekonstruktive soziologische oder technikhistorische Studien, sondern für eine entwicklungsnahe TA, denn solche Designphilosophien leiten die Modellierungsentscheidungen und damit die technische Spezifikation an.

\section{Ansatzpunkt 2: Forschungen zur „Human- Machine Interaction"}

Einen weiteren Ansatzpunkt eröffnet die Forschungscommunity der „Human-Machine Interaction“ bzw. hier einschlägiger: der „HumanRobot Interaction" (HRI), in der die Reaktion gewöhnlicher Menschen auf avancierte Roboter empirisch in Fallstudien untersucht wird. So hat etwa Scholtz (2003) verschiedene basale Rollen unterschieden, die Menschen intelligenten Robotern in ihrem Nahbereich zuschreiben.

In diesem Forschungsfeld herrscht durchgängig eine große Skepsis gegenüber der übergeneralisierten Annahme, dass ein freundliches Äußeres oder „menschenähnliches Verhalten“ von Robotern - besonders bei humanoiden Robotern - per se eine einfachere Interaktion mit diesen Maschinen sicher stellen kann:

„The criteria for 'good performance' often differ substantially. In particular, 'functionally designed' social robots may need only to produce certain experiences for the user, rather than having to withstand deep scrutiny for 'life-like' capabilities." (Steinfeld et al. 2006, S. 38)

Bei den Versuchen, die empirischen Ergebnisse der HRI zusammenzufassen, wird dann allerdings ein sehr allgemeines Verständnis von „Interaktion" zugrunde gelegt, und sämtliche möglicherweise relevanten Aspekte werden nur synoptisch aufgelistet. So präsentieren etwa Weiss et al. (2009) ein ,evaluation framework for Human-Robot Collaboration addressing usability, social acceptance, user experience, and societal impact", ergänzt um eine Mehrebenenindikatorik als Maß für die Akzeptanz und Sinnhaftigkeit von Roboteranwendungen. Dieses Bewertungsmaß ist zwar komplex, aber inhaltlich flach und statisch. Wenn dagegen von einem engeren Begriff der Interaktivität ausgegangen werden würde, dann könnte die Bewertung auf den Prozess zwischen Menschen und Robotern bezogen wer- 
den - als Prozess der wechselseitigen Anpassung oder als Verschränkung von genuin sozialem mit genuin maschinellem Lernen. Einer solchen hybriden Fassung der Fragestellung ist meines Wissens bislang noch nicht nachgegangen worden.

\section{Ansatzpunkt 3: Untersuchung der Mensch- Roboter-Interaktivität in realisierten Szenarien}

Ein weiterer viel versprechender Anknüpfungspunkt ist „Robocup“. Das sind Fußballturniere für Roboter, und die sind für weite Teile der Robotikszene das Ereignis schlechthin. Es ist der geniale Trick von Robocup, drei Organisationsprinzipien in einem Szenario zu vereinen. Das erste Prinzip ist ein rigides und nicht diskutierbares $\mathrm{Ma} ß$ für Erfolg - der Ball muss über die Torlinie. Das ist eine enorm schwierige Aufgabe für alle Teilkomponenten eines Roboters, und es gibt viele wissenschaftlich interessante Gründe für Misserfolge, aus denen die Entwickler sehr viel lernen können. Im Turnier selbst zählen diese Gründe, anders als in den Laboren, aber nicht. Das zweite Prinzip ist ebenso einfach: Die allseits bekannten Spielregeln von Fußball werden, nur leicht an Roboter angepasst, einfach übernommen. Das dritte Prinzip ist die Organisation einer gänzlich offenen Aushandlung des Wettkampf-Settings. Sowohl die Spezifikation des Spielfeldes wie die der Roboter und der Softwarearchitektur können von allen potenziellen Teilnehmern diskutiert werden, was in entsprechenden Mailingslisten auch eifrig geschieht. Nur in den wenigen Tagen des Turniers selbst sind die Regeln fixiert. In dieser Aushandlung gilt es, einen Kompromiss zwischen dem jeweiligen Entwicklungsstand in den beteiligten Disziplinen (von Fragen der Mechanik über Objekterkennung und Selbstlokalisierung bis zu Bewegungs- und Interaktionsplanung) einerseits und der Gewährleistung von Fair Play andererseits zu finden. Das hat inzwischen zur Differenzierung in mehrere verschiedene „Ligen“ geführt, die sich v.a. nach der Größe, dem Grad an Autonomie und der Gestalt der einzelnen Roboterspieler unterscheiden (mit Humanoiden als „Champions League“).

Für viele Forschende ist nun die Teilnahme an Robocup ein direkter Beitrag zur Entwicklung von Roboterassistenten für menschliche Alltagsumgebungen. Das erscheint kaum verwun- derlich, wenn berücksichtigt wird, dass zentrale Komponenten eines Fußballroboters auch für einen Serviceroboter notwendig sind, und diese Komponenten im Alltag - wie beim Fußballspiel - in „real time“ zusammen funktionieren müssen. Die Annahme auf Entwicklerseite ist auch hier, dass speziell humanoide Roboter in Aussehen und Verhalten Menschen ähneln und damit die Akzeptanz dieser Kreaturen steigern - eine Annahme, die auf keinerlei empirischer Evidenz gründet.

Aber der Siegeszug von Robocup hat inzwischen auch die Spezifik der Anwendungsdomäne menschliche Alltagsumgebungen erreicht. Mit „Robocup@home“ ist vor einigen Jahren eine neue Liga für menschliche Wohnumgebungen gestartet (http://www.robocupathome.org). In dieser Liga werden die gleichen Prinzipien wie im Fußballwettkampf verwendet. Das Spielfeld besteht aus einer stilisierten Wohnung mit mehreren Zimmern, Türen, und menschlichen Probanden, mit denen der Roboter interagieren muss. Das Maß für Erfolg ist die Abarbeitung einer aufeinander aufbauenden Kette von Teilaufgaben in einer definierten Zeit. Eine solche Kette könnte beispielsweise so aussehen: Gehe durch die Tür in die Wohnung, identifiziere eine menschliche Probandin, frag nach ihrem Namen, gehe in die Küche, identifiziere eine Flasche und greife sie, finde zurück zu der vorher identifizierten Probandin, reiche ihr die Flasche, und verlasse die Wohnung wieder. Für das Erreichen jeder dieser Teilaufgaben gibt es eine in den Spielregeln festgelegte Punktzahl. Und diese Spielregeln werden offen ausgehandelt wie bei den Fußball spielenden Robotern.

Nun hat Robocup@home ersichtlich nicht den Grad an nichtdiskutierbarer Evidenz des Maßes für Erfolg wie beim Fußball (der Ball muss hinter die Linie), und die Bewertung ist nicht ebenso rigide. Deshalb hat etwa der Schiedsrichter einen deutlich größeren Ermessenspielraum bei der Punktvergabe. Zudem ist Fairplay im Geiste eines tatsächlich entwicklungsrelevanten Wettbewerbs schwieriger abzusichern, weshalb es in den Regeln explizit heißt: ,trying to cheat (e.g. pretending autonomous behavior where there is none) and (...) trying to exploit the rules (e.g. not trying to solve the task but trying to score)" sind nicht erlaubt und werden, wenn das ,auffliegt“", bestraft (RoboCup@Home Technical Committee 
2009, S. 16). Besonders auffällig aber ist, dass die Probandinnen angewiesen werden, still zu halten und nur das zu tun bzw. zu sagen, was der Roboter auch „verstehen“ kann, obwohl das Ganze ausdrücklich darauf zielt, eine ,natürliche MenschRoboter-Interaktion “ zu befördern (dass., S. 5). In diesem Setting wird untersucht, oder besser: ,gemessen“, wie gewöhnliche Menschen aufComputer reagieren. Dies geschieht auf eine Weise, die ohne Zweifel entwicklungsrelevant ist. Aber es besteht keinerlei Austausch mit den Forschungen der komplementären Forschungscommunity der Human-Machine-Interaction oder der TA, obwohl das aus meiner Sicht nahe liegen würde.

\section{Fazit}

Roboethics und die Perspektive der Roboterentwickler erwecken zunächst den Eindruck zweier entgegengesetzter Lager, die sich an zwei entgegengesetzten Versionen der „Human Frontier“ der Mensch als ultimative Herausforderung oder der Roboter als Gefahr - orientieren. Anschließend an die Techniksoziologie habe ich für eine balanciertere Sichtweise plädiert, die auch das Potenzial hat, sich konkret in den Entwicklungsprozess einbringen. Das ist möglich, weil es auch auf Entwicklerseite anschlussfähige Forschungsund Diskussionsstränge gibt. Anstatt übergeneralisierte Annahmen über „den Menschen“ in Anschlag zu bringen, sollten die konzeptionellen Beiträge der Soziologie genutzt und empirische Untersuchungen der Mensch-Roboter-Interaktivität unternommen werden. Derzeit sind jedoch eine intensivere Kommunikation zwischen Roboterentwicklern und zu dem Gegenstand empirisch arbeitenden Soziologen und weitergehende Kooperationen im Sinne des Constructive TA vor allem noch eines: Desiderata.

\section{Anmerkungen}

1) Vgl. zu dieser in der Robotik üblichen Einteilung etwa Kawamura et al. 1996.

2) Wie bei Veruggio, Operto 2008, S. 1512.

3) Vgl. als ein Beispiel einer solchen Modellierung Häussling 2009.
4) Für Constructive Technology Assessment in der EU siehe Schot, Rip 1996, für Real-time Technology Assessment in den USA siehe Guston, Sarewitz 2002.

5) Brooks 1999; siehe auch Mitchell et al. 2000.

6) Veruggio, Operto 2008, S. 1522; vgl. auch Murphy 2000, S. 442.

\section{Literatur}

Akrich, M., 1995: User Representations: Practices, Methods and Sociology. In: Rip, A.; Misa, T.J.; Schot, J. (Hg.): Managing Technology in Society: The Approach of Constructive Technology Assessment. London, S. 167-184

Brooks, R.A., 1999: Cambrian intelligence: The Early History of the New AI. Cambridge, MA

Fong, T.; Nourbakhsh, I.; Dautenhahn , K., 2003: A Survey of Socially Interactive Robots. In: Robotics and Autonomous Systems 42 (2003), S. 143-166

Guston, D.H.; Sarewitz, D., 2002: Real-Time Technology Assessment. In: Technology in Society 24 (2002), S. 93-109

Häussling, R., 2009: Video Analysis with a four-level Interaction Concept: A network-based Concept of Human-Robot Interaction. In: Kissmann, U.T. (Hg.): Video Interaction Analysis. Methods and Methodology. Frankfurt a. M., S. 107-131

Kawamura, K.; Pack, T.; Bishay, M.; Iskarous, M., 1996: Design Philosophy for Service. In: Robots. Robotics and Autonomous Systems 18 (1996), S. 109-116 Meister, M.; Lettkemann, E., 2004: Vom Flugabwehrgeschütz zum niedlichen Roboter. Zum Wandel des Kooperation stiftenden Universalismus der Kybernetik. In: Strübing, J.; Schulz-Schaeffer, I.; Meister, M.; Gläser, J. (Hg.): Kooperation im Niemandsland. Neue Perspektiven auf Zusammenarbeit in Wissenschaft und Technik. Opladen, S. 105-136

Mitchell, R.J.; Bischop, J.M.; Keating, D.A.; Dautenhahn, K., 2000: Cybernetic Approaches to Artificial Life. In: Künstliche Intelligenz 14 (2000), S. 5-11

Murphy, R.R., 2000: An Introduction to AI Robotics. Cambridge, MA

Oudshoorn, N.; Pinch, T., 2007: User-Technology Relationships: Some Recent Developments. In: Hackett, E.; Amsterdamska, O.; Lynch, M.; Wajcman, J. (Hg.): The Handbook of Science and Technology Studies. Boston; MA, S. 541-567

Rammert, W.; Schulz-Schaeffer, I. (Hg.), 2002: Können Maschinen handeln? Soziologische Beiträge zum Verhältnis von Mensch und Technik. Frankfurt a. M.

Rammert, W., 2008: Where the Action is: Distributed Agency Between Humans, Machines, and Programs. In: Seifert, U.; Kim, J. H.; Moore, A. (Hg.): Para- 
doxes of Interactivity. Perspectives for Media Theory, Human-Computer Interaction, and Artistic Investigations. Bielefeld, S. 62-91

RoboCup@Home Technical Committee, 2009: Rules \& Regulations. Final Version; http://www.ai.rug.nl/ robocupathome/documents/rulebook2009 FINAL. pdf (download 29.6.09)

Scholtz, J., 2003: Theory and Evaluation of Human Robot Interactions. In: Proceedings of the 36th Hawaii International Conference on System Science; http://www.hicss.hawaii.edu/HICSS36/HICSSpapers/ETACH01.pdf (download 4.7.09)

Schot, J.; Rip, A., 1996: The Past and Future of Constructive Technology Assessment. In: Technological Forecasting and Social Change 54 (1996), S. 251-268

Siciliano, B.; Khatib, O. (Hg.), 2008: Handbook of Robotics. Berlin

Steinfeld, A.; Fong, T.; Kaber, D. et al., 2006: Common metrics for human-robot interaction. In: First ACM International Conference on Human Robot Interaction, Salt Lake City, UT, S. 33-40

Veruggio, G.; Operto, F., 2008: Roboethics: Social and Ethical Implications of Robotics. In: Siciliano, B.; Khatib, O. (Hg.): Handbook of Robotics. Berlin, S. 1499-1524

Wallach, W., 2010: Robot Minds and Human Ethics: The Need for a Comprehensive Model of Moral Decision Making. In: Ethics and Information Technology 12 (2010), S. 243-225

Weiss, A.; Bernhaupt, R.; Lankes, M.; Tscheligi, M., 2009: The USUS Evaluation Framework for HumanRobot Interaction. In: AISB2009: Proceedings of the Symposium on New Frontiers in Human-Robot Interaction. SSAISB, S. 158-165

Weiss, A.; Igelsböck, J.; Tscheligi, M. et al., 2010: Robots Asking for Directions - The Willingness of Passers-by to Support Robots. In: 5th ACM;IEEE International Conference on Human-Robot Interaction. Osaka, S. 23-30

Weyer, J., 2008: Techniksoziologie. Genese, Gestaltung und Steuerung soziotechnischer Systeme. Weinheim

\section{Kontakt}

Martin Meister

Technische Universität Berlin

Zentrum Technik und Gesellschaft

Sekr. ER 2-2

Hardenbergstraße 36 A, 10623 Berlin

E-Mail: meister@ZTG.tu-berlin.de

\section{Waffensysteme als „,derivier- te Akteure": Kampfroboter im asymmetrischen Krieg}

\author{
von Hans Geser, Universität Zürich \\ „, They don't forget their orders. “ \\ (P.W. Singer)
}

\begin{abstract}
Neueste Entwicklungen in der (semi-)autonomen Waffentechnik haben die (ursprünglich von I. Asimov entworfene) Idee des „Roboterkrieges" wiederbelebt. Funktionale Analysen zeigen, dass solche Geräte vielerlei für Menschen ungeeignete Spezialaufgaben übernehmen können, das offensive und defensive Potenzial der Streitkräfte verstärken und evtl. auch die Schwelle zum Gewalteinsatz niedriger legen. Andererseits ist ihr Nutzen aufgrund ihrer Gebundenheit an hochstrukturierte Umweltbedingungen und voraussehbare Problemtypen in asymmetrischen Kriegen (besonders in Friedensmissionen) eng begrenzt. Zudem erhöhen sie das Risiko, dass attackierte Feinde Zugang zu relevanten Informationen gewinnen. In dem Maße, wie Kampfroboter konventionelle Soldaten ersetzen, werden asymmetrische Gegner motiviert sein, ihre Aggression weg vom Schlachtfeld auf terroristische Aktivitäten zu verlagern.
\end{abstract}

\section{Von der bloßen Hand zum „derivierten Akteur"}

Am Ursprung der kriegstechnischen Evolution steht der unbewaffnete Mensch, der mit seinen bloßen Händen kämpft und damit alle motorischen und sensorischen Vorgänge mit seinem eigenen Körper kontrolliert. Seither hat sich die Waffentechnik fast ausschließlich darauf ausgerichtet, die motorischen Aspekte dieses Regelkreises (in Form von Steinen, Speeren, Wurfgeschossen, Artilleriegranaten und Interkontinentalraketen) zu externalisieren: mit der Konsequenz, dass die Zusammenhänge zwischen dem, was Kämpfer tun, und dem, was sie bewirken, immer vermittelter werden. Da die sensorischen wie auch die reflexiv-beurteilenden Prozesse vollumfänglich beim Menschen verbleiben, rückt der 